\title{
The Potential of N-Heterocyclic Carbene Complexes as Components for Electronically Active Materials
}

\author{
Oliver Schuster§a, Laszlo Mercs ${ }^{\mathrm{a}}$, and Martin Albrecht*ab \\ §SCS-DSM Nutritional Products Award Winner (Poster Presentation)
}

\begin{abstract}
The application of $\mathrm{N}$-heterocyclic carbene complexes as active sites in materials other than catalysis has been remarkably scarce. Inspired by the - often misleading - 'carbene' label, which implies a substantial degree of $\mathrm{M}=\mathrm{C} \pi$ bonding, we have been interested in evaluating the potential of $\mathrm{N}$-heterocyclic carbene complexes as building blocks for constructing electronically active materials. Electron mobility via the metal-carbon bond has been investigated in monometallic imidazol-2-ylidene complexes and subsequently expanded to polymetallic systems. In particular, ditopic benzobisimidazolium-derived ligands have been explored for the fabrication of bimetallic molecular switches and main-chain conjugated organometallic polymers. Electrochemical analyses have allowed the degree of electronic coupling between the metal sites to be quantified and the key parameters that govern the intermetallic communication to be identified.
\end{abstract}

Keywords: Electrochemistry · Functional materials · Molecular switches · N-heterocyclic carbenes · Organometallic polymers

\section{Introduction}

The area of molecular electronics has thus far been dominated by either organic or purely inorganic materials. For example, metallic GaAs and GaP nanowires have been constructed for semiconductor applications. ${ }^{[1]}$ While these materials show excellent electron mobility properties, their tuning potential is limited. In contrast, organic semiconductors such as conjugated oligomers or polymers display promising properties, ${ }^{[2]}$ though their electron carrier characteristics are intrinsically lower than those of metallic materials. Hybrid organic-inorganic materials might overcome these limitations and combine the advantages of purely inorganic and organic

${ }^{*}$ Correspondence: Prof. Dr. M. Albrecht ${ }^{\mathrm{ab}}$

Tel.: +35317162504

Fax: +353171625 01

E-mail: martin.albrecht@ucd.ie

aDepartment of Chemistry

University of Fribourg

Chemin du Musée 9

$\mathrm{CH}-1700$ Fribourg

bchool of Chemistry \& Chemical Biology

University College Dublin

Belfield

Dublin 4, Ireland

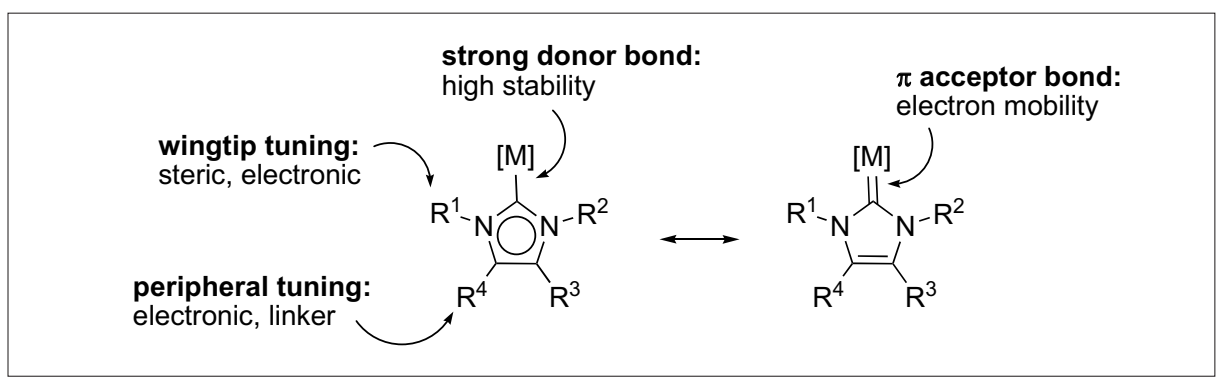

Fig. 1. Versatility of imidazolylidene complexes including partial $\pi$ bonding.

materials. Organometallic complexes may become especially useful as they comprise tunable organic ligand moieties linked by relatively strong $\mathrm{M}-\mathrm{C}$ bonds to transition metal centers. An appropriate choice of the metal center, e.g. redox-active iron or cobalt, is expected to enhance electron mobility. Moreover, the accessibility of different oxidation states of such metal centers may provide an additional switching function.

As ligands, we considered imidazolium-derived N-heterocyclic carbenes (NHCs) to be particularly promising, as the potential for $\mathrm{M}=\mathrm{C}$ carbene-type $\pi$ bonding may further stimulate electronic communication between the metal center and remote donor/acceptor sites located at the ligand periphery (Fig. 1). In addition, the strong donor ability and covalent character of the NHC-metal bond should provide further advantages for the fabrication of materials, such as increased stability of the coordinated metal center.

\section{Relevance of $\pi$ Backbonding in NHC Complexes}

The iron(II) complexes 1 and 2 (Fig. 2) have been synthesized to compare the donor properties of NHC ligands to those of related pyridines by analysis of the $\mathrm{Fe}^{\mathrm{II}} /$ Fe $^{\text {III }}$ redox potential. ${ }^{[3]}$ Through such electrochemical analysis, the ligand donor properties may be parametrized according to Lever electronic parameters (LEPs) ${ }^{[4]} \mathrm{A}$ LEP $E_{\mathrm{NHC}}=0.29$ has been deduced for the carbene ligand in complex 2 , which is only slightly higher than for pyridine $\left(E_{\text {pyridine }}=\right.$ $0.25)$. Determination of pertinent ligand LEPs is complementary to the more frequently used Tolman electronic parametrization, which relies on the energy of stretch vibrations of ancillary $\mathrm{CO}$ ligands. ${ }^{[5]}$ Moreover, LEPs seem to be less sensitive to stereoelectronic effects in the metal coordination sphere than Tolman electronic parameters (TEPs), though generally, 


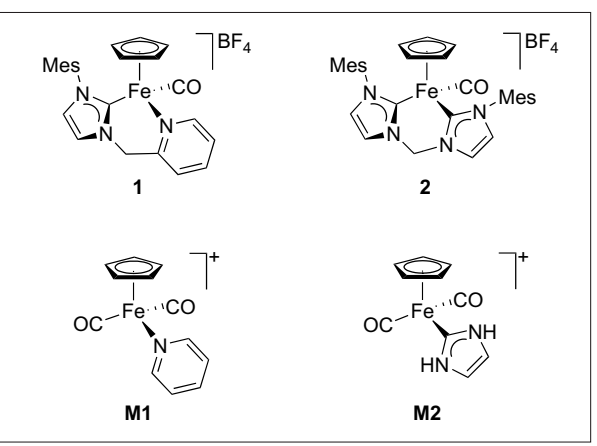

Fig. 2. Synthesized complexes $(\mathbf{1}, \mathbf{2})$ and theoretically investigated model complexes (M1, M2) for the comparison of imidazol-2ylidene ligands with pyridine.

TEPs and LEPs provide a consistent ligand donor scale. ${ }^{[6]}$

The similar LEP for $\mathbf{1}$ and $\mathbf{2}$ is surprising, especially when considering the strong $\sigma$ donor properties generally assumed for NHC ligands. Hence, a better stabilization of the +3 oxidation state and thus a significantly lower oxidation potential would be expected for the stronger donating ligand (cf. $E_{1 / 2}=+1.15$ for 1 and $E_{1 / 2}=+1.18$ for 2). The strongly related oxidation potentials indicate a similar electronic configuration of the metal centers and thus similar overall donor properties of the NHC and pyridine ligands in these complexes. Since $\sigma$ donation of carbenes is generally accepted to be stronger than for pyridines, ${ }^{[7]}$ our results suggest that also $\pi$ backbonding must be stronger in order to balance the net electron donor ability. Such a rationale is particularly relevant when considering that $\pi$ backdonation is well accepted for pyridine ligands. ${ }^{[8]}$ DFT calculations on the basis of the model compounds M1 and M2 (Fig. 2) predict relative $\pi$ contributions to the total orbital interactions of $17.8 \%$ and $15.4 \%$, respectively. These values support the experimental results and underline the relevance of $\pi$ bonding in these carbene complexes.

Such conclusions are corroborated by the analytical data for the rhodium(I) complexes 3 and 4 (Fig. 3). In an elegant approach, Bielawski and coworkers independently established metal-carbene $\pi$ interactions by comparing the quinone $\mathrm{C}=\mathrm{O}$ stretch vibrations and the reduction potentials. ${ }^{[9]}$ With two carbonyl groups formally conjugated with the carbene $\pi$ system, stretching frequencies were anticipated to respond to minute changes in the ligand $\pi$ system. Moreover, paraquinones exhibit reduction potentials that are sensitive to electronic changes at remote sites. In agreement with the weaker $\pi$ acidity of 1,5-cyclooctadiene (COD) as compared to $\mathrm{CO}$, a stronger $\pi$ backdonation has been deduced for complex

\begin{tabular}{|c|c|c|c|}
\hline & $\stackrel{O}{\|}$ & $\prod_{0}^{1}$ & Mes \\
\hline $\mathrm{C}=\mathrm{O}\left[\mathrm{cm}^{-1}\right]$ & 1670 & 1680 & \\
\hline$E_{\text {red }}[V]$ & -0.55 & -0.49 & \\
\hline
\end{tabular}

Fig. 3. Quinoneannelated imidazolylidene complexes with key IR and electrochemical data.

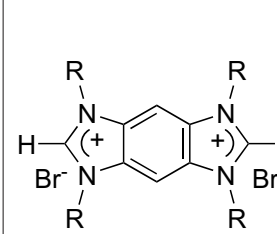<smiles>CCCCCCCC</smiles><smiles>[R][Y]1N([R])c2cc3c(cc2N1[R])N([R])[Y4]N3[R]</smiles>

3 based on the pertinent IR and electrochemical data.

\section{Coordination Polymers Based on Benzobisimidazolylidene Linkers}

Since these fundamental studies have revealed a certain degree of $M=C \pi$ interaction, Bielawski and coworkers have synthesized a variety of main-chain conjugated organometallic polymers (COMP) comprising rigid ditopic biscarbene linkers, such as benzobisimdazolylidenes 6 (Scheme 1). ${ }^{[10-12]}$ These organic building blocks offer two metal binding sites which are mutually connected via a conjugated $\pi$ system. Due to the mutual trans arrangement of the binding sites, bimetallic complexes are conveniently accessible.

The corresponding polymeric palladium and platinum materials poly-7 have been prepared by a $\mathrm{C}-\mathrm{H}$ bond activation protocol from the bisimidazolium salt $\mathbf{5}$ (Scheme 1). They are stable towards air and moisture. Their synthesis hence does not require anhydrous conditions or inert atmosphere, ${ }^{[10]}$ in contrast to metallations involving the free biscarbene 6.

Thin films of poly-7 casted onto silicon wafers show conductivities in the order of $10^{-3} \mathrm{~S} \mathrm{~cm}^{-1} \cdot{ }^{111]}$ For comparison, semiconducting silicon is about $100 \times$ less conductive $\left(10^{-5} \mathrm{~S} \mathrm{~cm}^{-1}\right)$. Moreover, these polymers are structurally dynamic and have therefore been tested as 'self- healing' materials. Successful healing of microcracks has indeed been observed, however, the presence of DMSO vapor appears to be essential for the healing process.

Notably, the bisimidazolium salt $\mathbf{5}$ does not afford stable polymeric materials when reacted with nickel(II) precursors. ${ }^{[10]}$ This result has been attributed to the reduced affinity of nickel for carboligands. ${ }^{[13]}$ However, nickel-containing coordination polymers poly- $\mathbf{8}$ have been readily obtained upon introducing a chelating phenolate wingtip group (Fig. 4). [12] The analogous palladium- and platinumcontaining polymers display an increased thermal stability compared to their nonchelated congeners poly-7. These results emphasize the strength of Bielawski's

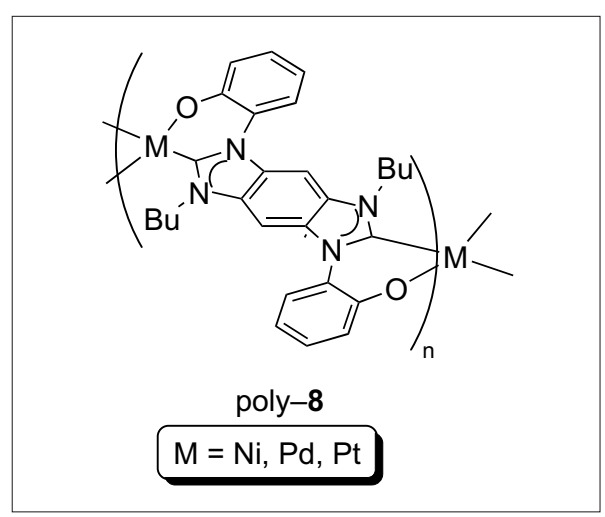

Fig. 4. Increased stability of COMPs through chelating wingtip groups. 
modular synthetic approach for N-substitution in the ligand precursor. In addition, they illustrate the tunability potential of NHC ligands through a wide variety of wingtip and arene substitution.

\section{Homobimetallic \\ Benzobisimidazolylidene Complexes}

Inspired by the versatility of such ditopic ligands, we have further investigated their potential for transferring electronic information between metal centers. ${ }^{[14]}$ Double metallation of the biscarbene precursor 9 with redox-active iron(II) centers has been accomplished via the free-carbene route to yield complex $\mathbf{1 0}$ (Scheme 2). ${ }^{[3]}$ The analogous dinuclear ruthenium(II) complex $\mathbf{1 1}$ has been prepared by transmetallation from the corresponding silver(I) complex. ${ }^{[15]}$ For comparative purposes, also the monometallic complexes 13 and $\mathbf{1 4}$ have been synthesized by identical methods starting from imidazolium salt 12 (Scheme 2).
Cyclic voltammetry (CV) measurements indicate that complex $\mathbf{1 0}$ undergoes a single reversible oxidation with $\mathrm{E}_{1 / 2}=$ $+0.54 \mathrm{~V}$ (Table 1). This potential compares well with the redox potential of the monometallic carbene complex 13 and indicates a metal- rather than a ligandcentered redox process. Differential pulse voltammetry (DPV) reveals that the oxidation signal in $\mathbf{1 0}$ is significantly broader than that of the monometallic species $\mathbf{1 3}$. Signal deconvolution using the DPV curve of the monocarbene complex as reference yields two oxidations that are separated by about $\Delta E_{1 / 2}=80 \mathrm{mV}$. This separation of the oxidation potentials corresponds to a comproportionation constant $\mathrm{K}_{\mathrm{c}}=22.5$ and reflects a weak intermetallic interaction. According to the Robin and Day classification, ${ }^{[16]}$ complex $\mathbf{1 0}$ represents a class II system and hence a molecular switch with two redox sites that are mutually dependent, albeit only weakly.

In contrast, the dinuclear ruthenium system 11 exhibits a considerably sharper oxidation at $\mathrm{E}_{1 / 2}=+1.18 \mathrm{~V}$. Experiments using DPV reveal only a small broadening
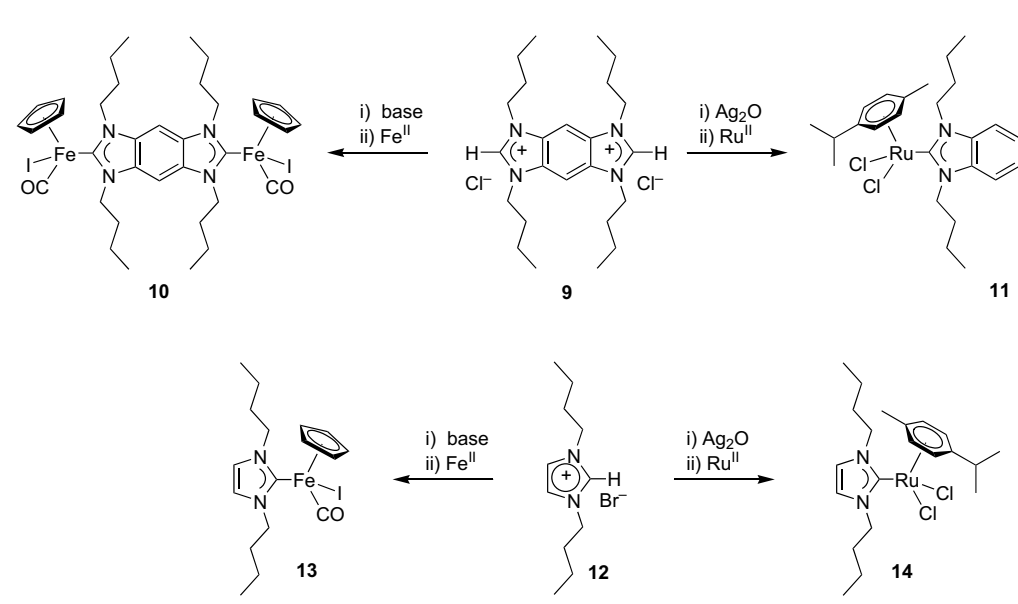

Scheme 2. Synthetic protocol towards homobimetallic molecular switches containing an NHC bridging ligand and their corresponding monometallic complexes.

Table 1. Electrochemical data for homobimetallic and monometallic NHC complexes.

$\begin{array}{lllll}\text { complex } & \text { metal fragment } & \text { ligand system } & E_{1 / 2}[\mathrm{~V}]^{\mathrm{b}} & \Delta \mathrm{E}_{1 / 2}[\mathrm{mV}]^{\mathrm{c}} \\ 10 & \mathrm{Fe}(\mathrm{cp})(\mathrm{CO}) \mathrm{I} & \mathrm{BBI} & +0.50,+0.58 & 80 \\ 11 & \mathrm{Ru}(\mathrm{cym}) \mathrm{Cl}_{2} & \mathrm{BBI} & +1.15,+1.21 & 58 \\ 13 & \mathrm{Fe}(\mathrm{cp})(\mathrm{CO}) \mid & \mathrm{In} \mathrm{Bu} & +0.42 & - \\ 14 & \mathrm{Ru}(\mathrm{cym}) \mathrm{Cl}_{2} & \mathrm{In} \mathrm{Bu} & +1.09 & - \\ 16 & \mathrm{Ir}(\mathrm{COD}) \mathrm{Cl} & \mathrm{BBI} & +0.91,+0.97 & 60 \\ 17 & \mathrm{Ir}(\mathrm{ppy})_{2} & \mathrm{BBI} & +0.81 & \text { n.d. } \\ 18 & \mathrm{Fe}(\mathrm{cp})(\mathrm{CO}) \mid & \mathrm{WT} & +0.58 & 80 \\ 19 & \mathrm{Fe}(\mathrm{cp})(\mathrm{CO}) \mid & \mathrm{WT} & +0.47 & \text { n.d. }\end{array}$

${ }^{\mathrm{a} B \mathrm{BI}}=$ benzobisimidazolylidene, $\mathrm{In}^{\mathrm{B}} \mathrm{Bu}=\mathrm{N}, \mathrm{N}^{\prime}$-di-n-butylimidazolylidene, $\mathrm{WT}=$ wingtip interlinked; bmeasurements in $\mathrm{CH}_{2} \mathrm{Cl}_{2}$ using $\left[\mathrm{NBu}_{4}\right]\left[\mathrm{PF}_{6}\right]$ as supporting electrolyte, potentials vs. SCE referenced to $\left[\mathrm{Ru}(\mathrm{bpy})_{3}\right]^{3+} /\left[\mathrm{Ru}(\mathrm{bpy})_{3}\right]^{2+}$ or $\mathrm{Fc}^{+} / \mathrm{Fc}$ as internal standard; ' determined by deconvolution of the signal from DPV, n.d. = not detectable. as compared to the related monometallic reference complex 14. Similar deconvolution provides two processes that are separated by only $58 \mathrm{mV}$. Accordingly, the ruthenium centers in $\mathbf{1 1}$ experience weaker mutual coupling than the iron centers in $\mathbf{1 0}$ and are essentially decoupled (class I system).

The small separation of the oxidation potentials hampered our efforts to stabilize and characterize any putative mixedvalent $\mathrm{M}^{\mathrm{II}}-\mathrm{M}^{\mathrm{III}}$ intermediate. Attempts to isolate the fully oxidized $\mathrm{M}^{\mathrm{III}}-\mathrm{M}^{\mathrm{III}}$ species have been equally unsuccessful due to the chemical instability of the fully oxidized product. Such instability becomes electrochemically apparent by the low reducing current upon exposing a sample of $\mathbf{1 1}$ at a potential slightly above $+1.2 \mathrm{~V}$ for prolonged time.

The poor intermetallic charge transfer ability of complexes $\mathbf{1 0}$ and $\mathbf{1 1}$ seems remarkable when assuming a supposedly fully conjugated $\pi$ system in the benzobiscarbene spacer ligand. Since the $\mathrm{M}-\mathrm{C}$ bond in NHC iron complexes has previously been shown to contain some $\pi$ character (see above), ${ }^{[3]}$ the weak coupling may be attributed to a limited conjugation between the heterocyclic NCN amidinylidene moiety and the central arene fragment of the ligand. Such a model is supported by the fact that the electronic properties of benzimidazolylidene ligands are more closely related to saturated imidazolinylidenes than to unsaturated imidazolylidene ligands, ${ }^{[17]}$ hence indicating a weak orbital overlap between the benzene fragment and the NCN unit in metal complexes. Alternatively, weak $\mathrm{d}_{\text {metal }}-\pi_{\mathrm{NHC}}$ orbital overlap due to an unfavorable geometrical arrangement of the ligand plane and the metal coordination system may prevent strong electronic coupling.

Bielawski and co-workers have noted a similar behavior for the bimetallic iridium(I) complex 16 (Scheme 3). ${ }^{[18]}$ Only moderate coupling has been deduced from DPV deconvolution, which is in good agreement with our own findings (Table 1). Remarkably, the chelated iridium(III) complex 17 comprising an $\operatorname{Ir}(\text { ppy })_{2}$ metal unit (ppy $=2$-phenylpyridyl) and a chelating biscarbene linker features essentially decoupled metal centers. An unfavorable geometrical metal-ligand arrangement may be excluded due to the C,C-bidentate chelation of the spacer ligand. Instead complete lack of orbital overlap has been proposed to originate from a too large energy difference between the interacting d-orbitals of the iridium(III) center and the ligand-centered $\pi$ acceptor MO. This energy difference is expected to be larger for iridium(III) in $\mathbf{1 7}$ than for the relatively electron-rich iridium(I) in 16. Accordingly, tailoring of the frontier orbitals should 


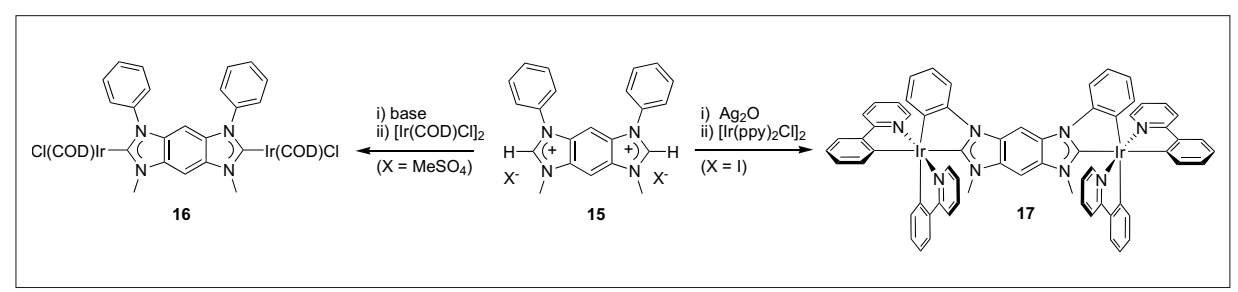

Scheme 3. Synthetic protocol towards bimetallic iridium-based molecular switches.

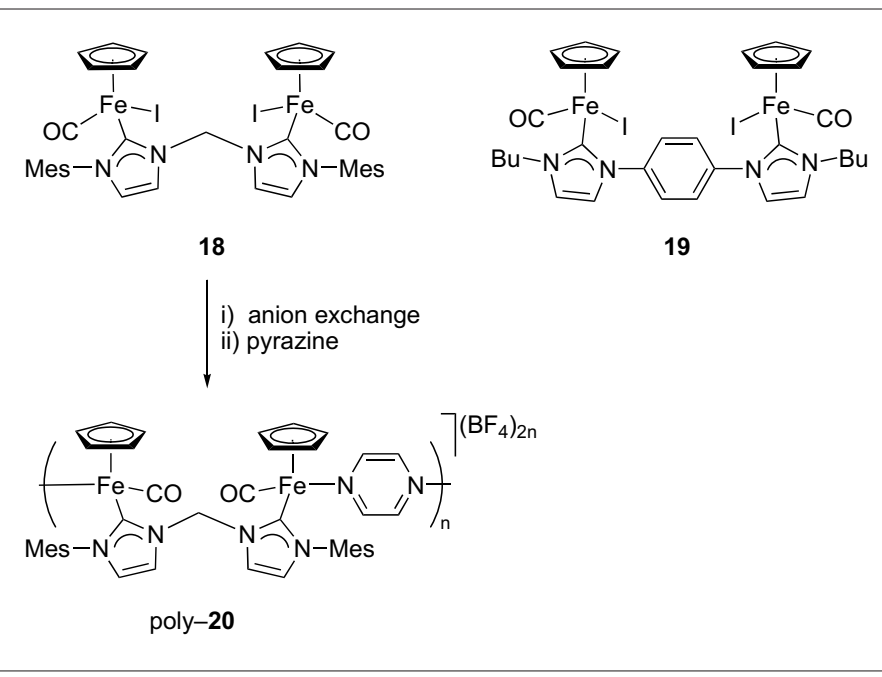

provide a methodology for increasing the intermetallic coupling. Besides modification of the metal oxidation state, clearly the type of metal (electron density, orbital energies) and the donor properties of spectator ligands will play a crucial role for maximizing orbital overlap.

\section{N-Bridged Bimetallic Iron(II) Complexes}

A different interconnection of the redox-active metal centers has been probed by using dicarbene ligand precursors that are interlinked at the heteroatom. A variety of spacers has been tested such as the methylene group in $\mathbf{1 8}$ and the phenylene unit in 19 (Scheme 4). [19] While dimetallic complexes such as $\mathbf{1 9}$ are readily accessible via the free carbene route, synthetic modifications have been implemented for the preparation of complex $\mathbf{1 8}$ in order to prevent chelation of the dicarbene ligand. [3] Strict control of the reaction temperature paired with a rigid addition protocol affords the bimetallic complex $\mathbf{1 8}$ in good yields.

Cyclic voltammetry measurements indicate a single reversible oxidation for both complexes 18 and 19 at $E_{1 / 2}=0.58 \mathrm{~V}$ and $0.47 \mathrm{~V}$, respectively. DPV signal deconvolution suggests metal-metal communication in the phenylene-bridged species $\mathbf{1 9}$ $\left(\Delta E_{1 / 2}=80 \mathrm{mV}\right)$.

Complexes 18 and 19 have been successfully employed as building blocks in main-chain organometallic polymers. In
Scheme 4. Synthesis of a representative mainchain organometallic polymer (poly-20) from heteroatom-interlinked dinuclear iron(II) complexes.

ular when considering the versatility of the NHC ligand for functionalization and the numerous methods that are available for installing the metal center. As a next challenge, a profound understanding on how to maximize intermetallic communication needs to be established. These efforts will encompass variation of the metal fragment (e.g. type of metal center, oxidation state, coordination geometry, heteronuclear systems) as well as modulation of the ligand framework (e.g. chelation, steric demand, type of carbene-interlinking moiety). Given this high optimization potential, it is likely that metal carbene synthons will provide interesting components for application in molecular electronics and for preparing new functional materials.

\section{Acknowledgements}

Our research in this area has been supported by the Swiss National Science Foundation, the European Marie Curie Action (Intra-European Fellowship to O.S.) and the Alfred Werner Foundation (Assistant Professorship to M.A.).

Received: January 8, 2010

[1] M. S. Gudiksen, L. J. Lauhon, J. Wang, D. C Smith, C. M. Lieber, Nature 2002, 415, 617.

[2] N. C. Greenham, S. C. Moratti, D. D. C. Bradley, R. H. Friend, A. B. Holmes, Nature 1993, 365, 628 .

the presence of a ditopic imine ligand such as pyrazine or 4,4'-bipyridine, polymerization ensues. While the bipyridine-bridged systems seem to feature electronically isolated metal centers, broad oxidation and reduction signals have been observed for poly-20 comprising pyrazine linkers. Such behavior has been attributed to multimetallic interactions and is characteristic for an electronically conducting molecular wire. A drawback of this prototypical system consists, however, of the restricted stability of poly-20 in solution, presumably due to the weak coordination of the pyrazine. Work directed towards increasing the stability of the imine-metal bond is currently in progress.

\section{Conclusions and Outlook}

The application potential of metal carbene complexes in electronically active functional materials is largely determined by the degree of electron mobility that can be achieved with these kinds of building blocks. Initial studies have indicated appreciable electronic coupling via the metal-carbon bond in certain cases, though they have also unveiled a high sensitivity towards small variations in the metal's electronic configuration. Inefficient overlap of the interacting orbitals at the metalligand boundary as well as reduced delocalization within the ditopic linkers have been identified as key factors. These issues are expected to be solved readily, in partic-
[3] L. Mercs, G. Labat, A. Neels, A. Ehlers, M Albrecht, Organometallics 2006, 25, 5648.

[4] a) A. B. P. Lever, Inorg. Chem. 1990, 29, 1271 b) A. B. P. Lever, Inorg. Chem. 1991, 30, 1980.

[5] C. A. Tolman, Chem. Rev. 1977, 77, 313.

[6] L. Perrin, E. Clot, O. Eisenstein, J. Loch, R. H Crabtree, Inorg. Chem. 2001, 40, 5806.

[7] E. F. Penka, C. W. Schlaepfer, M. Atanasov, M Albrecht, C. Daul, J. Organomet. Chem. 2007, 692, 5709.

[8] a) L. F. Lindoy, S. E. Livingstone, Coord. Chem. Rev. 1976, 2, 173; b) A. P. Smith, C. L. Fraser in 'Comprehensive Coordination Chemistry II', Eds. J. A. McCleverty, T. J. Meyer, Elsevier, Oxford, 2004, p. 1

[9] M. D. Sanderson, J. W. Kamplain, C. W Bielawski, J. Am. Chem. Soc. 2006, 128, 16514.

[10] A. J. Boydston, K. A. Williams, C. W. Bielawski, J. Am. Chem. Soc. 2005, 127, 12496

[11] K. A. Williams, A. J. Boydston, C. W. Bielawski, J. R. Soc. Interface 2007, 4, 359.

[12] A. J. Boydston, J. D. Rice, M. D. Sanderson, O. L. Dykhno, C. W. Bielawski, Organometallics 2006, 25, 6087 .

[13] J. Louie, J. E. Gibby, M. V. Farnworth, T. N Tekavec, J. Am. Chem. Soc. 2002, 124, 15188.

[14] L. Mercs, A. Neels, M. Albrecht, Dalton Trans. 2008, 5570 .

[15] a) H. M. J. Wang, I. J. B. Lin, Organometallics 1998, 17, 972; b) A. R. Chianese, X. W. Li, M. C. Janzen, J. W. Faller, R. H. Crabtree, Organometallics 2003, 22, 1663.

[16] M. B. Robin, P. Day, Adv. Inorg. Chem. Radiochem. 1967, 10, 247.

[17] F. E. Hahn, M. C. Jahnke, Angew. Chem., Int. Ed. 2008, 47, 3122.

[18] A. G. Tennyson, E. L. Rosen, M. S. Collins, V. M. Lynch, C. W. Bielawski, Inorg. Chem. 2009 $48,6924$.

[19] L. Mercs, A. Neels, H. Stoeckli-Evans, M. Albrecht, Dalton Trans. 2009, 7168. 